\section{UNBIASED ASSESSMENT OF SYMPTOMATIC “RESPONSE RATE" TO CARDIAC RESYNCHRONISATION THERAPY BY SYSTEMATIC REVIEW OF RANDOMISED CONTROLLED TRIALS (REVERSE, MIRACLE, MIRACLE ICD, MIRACLE ICD II, CARE-HF, COMPANION, CONTAK-CD, AND MUSTIC)}

doi:10.1136/heartjnl-2012-301877b.1

S M A Sohaib, * Z Chen, Z Whinnett, D P Francis, C Manisty. Imperial College London, London, UK

Background Varied rates of individual symptomatic response are cited for cardiac resynchronisation therapy (CRT) but have never been systematically evaluated together. Nor has spontaneous recovery rate been routinely subtracted, to clearly identify rate of symptomatic response genuinely attributable to CRT.

Method and Results First, we systematically reviewed the last 92 papers on PubMed about CRT. 74\% referred to responder rates but only $18 \%$ recognised the existence of "response to doing nothing". Second, we examined symptomatic response rates in the randomised CRT trials CARE-HF, COMPANION, CONTAK-CD, MIRACLE, MIRACLE-ICD, MIRACLE-ICD II, MUSTIC, and REVERSE, totalling 3904 patients. The weighted average symptomatic response rate, assessed using the clinical composite score was $54 \%$ for those randomised to CRT vs $40 \%$ for those randomised to no CRT. Using NYHA score, these values were $51 \%$ and $35 \%$ respectively. When symptomatic response rate was measured using 6-min walk distance and Minnesota Living with Heart Failure Quality of Life Score, a much larger spontaneous improvement was seen in the control arm of the blinded studies (device implanted but turned off in the control arm) compared to the open studies (no device implanted in the control arm). Spontaneous improvement was almost twice as high in the control arms of the blinded studies vs the open studies. With 6-min walk distances, $55 \%$ of the improvement in distance walked in the CRT arm was seen in the control arm for the blinded studies, vs $25 \%$ in the open. These values were $56 \%$ and $23 \%$ respectively with the Minnesota Living with Heart Failure Quality of Life Score.

Conclusions Quoting CRT responder rates in isolation, without recognising spontaneous responders, is common but invalid. Response rate with CRT, at $54 \%$, is not the response rate attributable to CRT, which is only $14 \%$ of implanted patients. Threequarters of those who "responded" with CRT would have done so even without CRT. Subjective quantitative markers seem to show an additional placebo effect and the placebo effect is more pronounced in the blinded studies than open studies. CRT definitely prevents death and reduces symptoms, but commonly-quoted "responder rates" are exaggerated, and are dependent on the measure used, and the blinding methodology used in the trial referenced.

\section{A RANDOMISED STUDY OF TEMPORARY EPICARDIAL CARDIAC RESYNCHRONISATION VS CONVENTIONAL RIGHT VENTRICULAR PACING IN CARDIAC SURGICAL PATIENTS}

doi:10.1136/heartjnl-2012-301877b.2

${ }^{1} \mathrm{~S}$ J Russell, ${ }^{2} \mathrm{C}$ Tan, ${ }^{3} \mathrm{~S}$ Ashraf, ${ }^{3} \mathrm{~A}$ Zaidi, ${ }^{2} \mathrm{P}$ O'Keefe, ${ }^{1} \mathrm{~A}$ G Fraser, ${ }^{2} \mathrm{Z}$ R Yousef ${ }^{1}$ Wales Heart Research Institute, Wales, UK; ${ }^{2}$ University Hospital of Wales, Wales, UK; ${ }^{3}$ Morriston Hospital, Swansea, UK

Introduction Left ventricular (LV) function is an important indicator of morbidity and mortality after cardiac surgery. Therefore, interventions to optimise peri-operative LV function may improve surgical outcomes. Biventricular (BiV) pacing has been shown to improve haemodynamic function in heart failure patients and temporary BiV pacing is feasible after cardiac surgery. Therefore, temporary BiV pacing may be beneficial to heart failure patients after cardiac surgery.

Aim The aim of this trial was to investigate the clinical utility of temporary BiV pacing delivered via epicardial wires attached to the right atrium (RA), right ventricle (RV) and left ventricle (LV) after cardiac surgery.

Methods 55 subjects undergoing elective cardiac surgery (coronary artery bypass grafting and/or valve surgery) were recruited from two cardiac centres, over an 18-month period commencing January 2010. 38 subjects completed the protocol. Nine subjects were removed at the operators request including: "off pump" bypass surgery, patient referred for alternative mode of revascularisation or surgical "turn down". 19 subjects were randomly assigned to receive temporary $(\mathrm{BiV})$ pacing using a dedicated triple chamber temporary pacing box with the capacity to programme the atrio-ventricular (AV) and inter-ventricular (VV) intervals. 19 received "standard pacing" after cardiac surgery. The duration of level 3 care was measured for each subject. In brief, this is the requirement for either invasive ventilation, multi-organ support or haemodynamic support with more than one inotrope/vasocontrictor or intra-aortic balloon pump. The trial was powered to compare the primary endpoint of transition from level 3 to level 2 care in the two groups. Secondary endpoints included acute haemodynamic performance in different pacing modes: immediately after the operation, $6 \mathrm{~h}, 18 \mathrm{~h}$ and $24 \mathrm{~h}$ after admission to cardiac intensive care. The pacing modes assessed

Abstract 001 Figure 1 Weighted average improvement in 6-min walk distance and Minnesota living with heart failure score in control and therapeutic arms of CRT trails. 